(C) 2021, The Authors. Published by Elsevier Inc. and Fass Inc. on behalf of the American Dairy Science Association ${ }^{\circledR}$. This is an open access article under the CC BY-NC-ND license (http://creativecommons.org/licenses/by-nc-nd/4.0/).

\title{
Disbudding and dehorning practices for preweaned dairy calves by farmers in Wisconsin, USA
}

\author{
Julia Saraceni, ${ }^{1} \odot$ Charlotte B. Winder, ${ }^{1 *} \odot$ David L. Renaud, ${ }^{1}{ }^{\oplus}$ Cynthia Miltenburg, ${ }^{2} \odot$ Erin Nelson, ${ }^{3} \odot$ \\ and Jennifer M. C. Van Os ${ }^{4}$ \\ ${ }^{1}$ Department of Population Medicine, University of Guelph, Guelph, ON, Canada, N1G 2W1 \\ ${ }^{2}$ Ontario Ministry of Agriculture, Food and Rural Affairs, Guelph, ON, Canada, N1G 2W1 \\ ${ }^{3}$ Department of Sociology and Anthropology, University of Guelph, Guelph, ON, Canada, N1G 2W1 \\ ${ }^{4}$ Department of Animal and Dairy Science, University of Wisconsin-Madison, 53706
}

\section{ABSTRACT}

Many dairy farmers in North America disbud or dehorn their cattle to improve human and animal safety. The Farmers Assuring Responsible Management (FARM v. 4.0) program requires that disbudding be performed before 8 wk of age with pain-control medication. The objective of this observational cross-sectional study was to quantify disbudding and dehorning practices of Wisconsin dairy producers to target future extension programming. Responses from 217 Wisconsin dairy producers and calf raisers were collected via digital surveys distributed at extension events and through industry contacts. Of the 217 respondents, 188 performed on-farm disbudding themselves. Most respondents (61\%) used caustic paste as their primary method, which was most commonly applied on the day the calf was born (53\%). Hot iron was used by $32 \%$ of respondents, and surgical methods (gouge, scoop, or wire saw) were used by $6 \%$ of respondents. Hot-iron disbudding was most commonly performed at 4 to 8 wk of age (41\%) and 1 to 4 wk of age (33\%), whereas surgical methods were most commonly performed at 8 wk or older (73\%). Pain-control medication was used by $43 \%$ of respondents. Specifically, $35 \%$ used an anti-inflammatory, and 21\% used a local nerve block. Veterinary involvement in creating the disbudding protocol was associated with increased odds of using pain control. Respondents with a target weaning age of $\geq 10$ wk had greater odds of complying with FARM disbudding requirements and were also more likely to use polled genetics. Respondents aged 18 to 34 and respondents with $>60$ calves were more likely to have made changes to their disbudding or dehorning protocol in the last decade. Although use of pain control

Received March 5, 2021.

Accepted June 23, 2021.

*Corresponding author: winderc@uoguelph.ca was higher than in previous US studies, full adoption of pain management requires further extension efforts. Veterinarians appeared influential on adoption of pain control, and their involvement may encourage adoption of pain management. Further research should investigate how the implementation of new FARM v. 4.0 standards will change the disbudding and dehorning practices of American dairy producers.

Key words: pain management, welfare, dairy calf, analgesia, anesthesia

\section{INTRODUCTION}

Many farmers around the world choose to disbud or dehorn cattle to mitigate the risk of injury to themselves and other animals posed by horned cattle (Stock et al., 2013; Cozzi et al., 2015). Disbudding refers to the destruction of the horn-producing germinal cells of the horn bud of young calves (AVMA, 2014), and is often performed using caustic paste or a hot iron (USDA, 2018). Dehorning refers to removal of the horn once it has attached to the frontal sinus of the skull and requires more invasive amputation methods (CVMA, 2016).

All disbudding or dehorning methods are painful (Stafford and Mellor, 2005, 2011). Amputation (dehorning) produces the most dramatic increase in pain markers compared with disbudding methods (Stafford and Mellor, 2011; Stock et al., 2013). The initial serum cortisol spike, an indicator of acute pain and stress, is virtually eliminated through the use of a local anesthetic block, for all methods of disbudding and dehorning (Stilwell et al., 2009; Winder et al., 2018a,b; Reedman et al., 2020b). Longer term inflammatory pain has been shown to be reduced by administering a nonsteroidal anti-inflammatory drug (NSAID) for both caustic paste (Stilwell et al., 2009; Winder et al., 2017; Reedman et al., 2020b), and hot-iron methods (Stock et al., 2013; Winder et al., 2018b). Sedation may also be used during these procedures to help manage calf handling; 
however, the use of xylazine alone is insufficient at eliminating the pain response associated with disbudding (Stilwell et al., 2010). Although preliminary work has shown some benefit, there is a research gap with respect to how sedatives may work in conjunction with local anesthetics and NSAID to mitigate pain during disbudding or dehorning (Caray et al., 2015; Bates et al., 2016; Reedman et al., 2020a).

Many veterinary organizations and animal welfare assessment programs have statements regarding use of pain management when calves are disbudded or dehorned. Specifically, the Canadian Veterinary Medical Association (CVMA) and American Association of Bovine Practitioners (AABP) recommend the use of local anesthetic and analgesic drugs for all methods of disbudding or dehorning (CVMA, 2016; AABP, 2019). American dairy producers who participate in the Farmers Assuring Responsible Management (FARM) program have new expectations as of 2020 that state that a veterinarian must be involved in the creation of a disbudding or dehorning protocol, calves must be disbudded before 8 wk of age and pain-control medication must be used for all disbudding and dehorning practices (FARM, 2020). Specifically, the expectation for disbudding age has been elevated from a continuous improvement plan with a 3 -yr corrective timeline to a mandatory corrective action plan with a 9-mo corrective timeline. The expectations for pain-control use and veterinary involvement are new standards with a continuous improvement plan and a 3 -yr corrective timeline as of January 1, 2020 (FARM, 2020). Although participation in the FARM program is voluntary for American producers, it is often required by milk processors and is increasingly popular, with $99 \%$ of the US milk supply covered under the FARM program (National Dairy Farm Program, 2020).

Although previous studies conducted in 2004, 2005, and 2014 revealed that the majority of producers in the United States $(81 \%, 88 \%$, and $72 \%$ respectively) perform these procedures without any pain mitigation (Hoe and Ruegg, 2006; Fulwider et al., 2008; USDA, 2018), recent research exploring if these practices have changed since that time is lacking. A 2016 study investigating the disbudding and dehorning practices in Ontario, Canada showed that producer and veterinary use of local anesthetics, sedatives, and NSAID when disbudding and dehorning had substantially increased compared with work conducted 10 yr prior (Winder et al., 2016). Similarly, the use of NSAID for disbudding and dehorning by veterinarians in the United Kingdom was seen to have significantly increased when compared with previous estimates from 2006 (increased to $56 \%$ from 3\% previously; Hambleton and Gibson, 2017).
These results demonstrate that substantial progress can be made in the uptake of pain control for disbudding and dehorning over time.

The objective of this cross-sectional study was to understand current disbudding and dehorning practices performed by Wisconsin dairy producers to better target extension programming at adoption of best practices by producers. This study also explored producer demographics, farm demographics, and farmer attitudes, and their associations with different disbudding and dehorning practices. It was hypothesized that veterinarian involvement would be highly associated with use of pain-control medication, as well as compliance with FARM disbudding practices.

\section{MATERIALS AND METHODS}

This manuscript is reported following the Strengthening the Reporting of Observational Studies in Epidemiology - Veterinary Extension (STROBE-vet) reporting guidelines statement (Sargeant et al., 2016). This project was approved by the University of Wisconsin (UW)-Madison Education and Social/Behavioral Science Institutional Review Board (submission ID no. 2019-1000).

\section{Survey Populations and Survey Delivery}

The survey was distributed throughout the fall of 2019 at extension events within Wisconsin, as well as online (i.e., email, social media). Although any producer in the United States was eligible to participate, Wisconsin was the most commonly represented state. Wisconsin was specifically targeted for this survey due to it having the second highest volume of milk produced and number of dairy cows in the United States (USDA, 2019). A subanalysis specific to the disbudding and dehorning practices of Wisconsin dairy producers was planned. Survey participants were recruited through industry contacts (i.e., veterinarians, nutritionists, sponsors, industry organizations) and at producer extension events. The 2019 World Dairy Expo in Madison, Wisconsin was the main in-person event used for participant recruitment; however, this event did not result in a substantial number of respondents. Individual UW-Madison Division of Extension county agriculture educator team members also administered the survey at other events and in one-on-one interactions with producers.

Eligibility criteria of participants in the survey included English-speaking dairy producers or calf raisers in the United States. Participants were selected based on their availability and willingness to participate in 
the survey, including being at least 18 yr of age and able to answer questions about calf-management practices on the farm where they worked. The survey was closed on December 31, 2019, before implementation of new requirements for FARM (version 4.0). A total of 423 producers were surveyed from various states across the United States; however, only producers from Wisconsin who performed their own on-farm disbudding or dehorning (188) were included in this analysis. No formal sample size calculation was completed for this survey. A convenience sample of producers was used to reflect the population that would be likely to engage in extension programming hosted by UW-Madison. Researcher motivations were tied to extension work; therefore, the convenience sample was representative of those who were already interested in UW-Extension, as well as those who would be likely to participate in future extension events.

\section{Survey Design}

This survey was a mixed methods questionnaire, with both qualitative and quantitative aspects. Seventy-nine survey questions on disbudding and dehorning practices were developed based on questions used in previous surveys (Winder et al., 2016; A. Stanton, L. Binersie, and Z. Miller, University of Wisconsin-Madison Extension, unpublished data, 2015) and audience questions during UW-Madison extension presentations. The survey also explored other aspects of calf care, including calf housing, feeding, weaning, and respondent satisfaction with calf growth and performance (Supplemental File S1; https://doi.org/10.5683/SP2/BXNRES). Survey collaborators reviewed the questions before they were approved for distribution. The survey was piloted by 6 dairy producers to ensure appropriate survey length and clarity of the questions. Pilot responses were included in the final analysis because no substantial survey changes were made. The survey was hosted in Qualtrics (2019 version, https://www.qualtrics.com) and delivered directly to participants online (i.e., email, social media) and through the Qualtrics app, which was preloaded onto tablet computers (iPad Mini 5, Apple Inc.) at in-person extension events.

Although this survey was distributed to a convenience sample of producers, certain measures were taken to avoid biases through survey design. The presentation of multiple-choice responses was randomized whenever possible, and the number of questions presented on each page of the survey was limited to prevent the respondent from seeing how certain questions led to others. The survey was also advertised as a "calf-care survey" rather than a disbudding or dehorning survey.
Questions included those on demographics, including role on the farm. Respondents were classified as owners or co-owners, farm managers, or calf-care staff. Those who indicated other roles were categorized into the most similar category. Herd size was estimated using the reported number of heifer calves that were being fed milk at the time of the survey. Respondents were asked to indicate the percentage of their calves that were disbudded or dehorned with each of the following methods: caustic paste; small burner; larger burner; gouge, scoop, or Barnes method; wire saw; and other. Respondents were also able to select "I don't know" as a response to this question. Using a wire saw or gouge, scoop, or Barnes method was combined into 1 category as "surgical methods." Large and small burners were also combined into 1 category.

Respondents were asked how painful they perceived the following 5 different disbudding or dehorning scenarios to be (assuming no use of pain mitigation): caustic paste administered on d 1 of life, caustic paste administered on $\mathrm{d} 10$, hot iron on $\mathrm{d} 10$, hot iron at $7 \mathrm{wk}$, and gouge and hot iron at 5 mo. Respondents scored each method from 0 to 10, with 10 reflecting the worst pain imaginable and 0 reflecting no pain at all. These 5 scenarios were thought to represent the general calf ages at which these disbudding and dehorning practices are typically performed. The pain scores associated with disbudding calves at 7 wk old using a hot iron were used as an estimate of pain perception in logistic regression models. This method and age were thought to be familiar for most respondents, as most producers perform disbudding with a hot iron (USDA, 2018), and 7 wk was thought to be a common age for this procedure.

Respondents were also asked to indicate which medications they used for disbudding or dehorning the majority of the time, which included the following: a local nerve block, a sedative or tranquilizer, or an NSAID. Respondents could also select "no medications are used" or "I don't know." Respondents were asked how often they had to repeat disbudding or dehorning on their farm, as well as questions related to their veterinarian, such as if their veterinarian-producer relationships had influenced their disbudding or dehorning methods, if they had regularly scheduled visits with their veterinarian, and if their veterinarian was involved in developing their disbudding or dehorning protocol.

\section{Statistical Analysis}

Survey responses were exported from Qualtrics into Microsoft Excel (version 16.42 for Mac, Microsoft Corporation). Descriptive statistics, including count 
data, mean scores, median scores, standard deviations, maximum values, and minimum values, were evaluated in Excel, whereas STATA15 (Stata/IC version 15.1 for Mac, StataCorp) was used for all regression analysis. An a priori data analysis plan (Supplemental File S2; https://doi.org/10.5683/SP2/BXNRES) was created for all analyses, including strategies for regression model building and thematic analysis for the open text responses.

Participant responses to questions concerning producer role and primary disbudding or dehorning method were categorized for clarity and analysis. Respondents who were owners and had other roles on the farm were included in the "owner or co-owner" category. Herd managers, calf managers, and any respondents with a managerial role [i.e., consultant $(\mathrm{n}=$ $1)$, general manager $(n=1)$, veterinarian $(n=2)$ ] were categorized as "managers." Calf-care staff and general farm staff were classified as "calf-care staff." Respondents were categorized into their primary disbudding or dehorning method based on the method that they reported to use most commonly on their calves. Seven respondents had an equal split between 2 methods. For analytical purposes, these respondents were categorized into the disbudding or dehorning method with fewer respondents in order to increase the comparability of categories. Those reporting $50 \%$ surgical dehorning and $50 \%$ disbudding (hot iron or caustic paste; $\mathrm{n}=5$ ) were classified as primarily surgical. Two respondents reported 50\% hot iron and 50\% caustic paste and were categorized as primarily hot iron.

A logistic regression model was built for each of the following outcomes: respondent compliance with FARM v. 4.0 disbudding and dehorning expectations, respondent decision to make changes to their disbudding or dehorning protocols, respondent use of pain control, and respondent use of polled genetics. In each model, continuous variables were visually assessed for linearity using a Lowess curve and were adjusted to correct for a nonlinear result if needed. Univariate analyses were done for each model using a liberal $P$-value $(<0.20)$. Variables that were potentially significant at the univariate level were offered to the multivariable model. Significant variables at a $P$-value of 0.05 were retained in the final model. Two-way interactions were evaluated between variables suspected to interact based on evidence from the literature and remained in the final models if significant. Confounders were identified as any variable that when removed, changed the coefficients of the remaining variables by a value greater than $20 \%$. Outliers were visually evaluated using a scatter plot of standardized residuals and model fit was assessed using the Hosmer-Lemeshow goodness-of-fit test.
Differences in equality of distribution were tested for all 2-way comparisons between the 5 disbudding or dehorning scenarios (caustic paste applied on d 1, caustic paste applied on d 10, hot iron used on d 10, hot iron used at $7 \mathrm{wk}$, gouge and hot iron used at $5 \mathrm{mo}$ ) using Kolmogrov-Smirnov tests. Additionally, differences in equality of distribution between respondents categorized as primarily hot iron and caustic paste users were assessed within each of the 5 disbudding or dehorning scenarios using Kolmogrov-Smirnov tests. As surgical amputation was an uncommonly selected method, this was not evaluated. A critical value of $P<0.05$ was used for all tests, and histograms were used to visually assess differences in distributions between groups.

\section{RESULTS}

\section{Respondent Demographics}

Answering all questions in this survey was not a mandatory requirement; therefore, some questions will not have a $100 \%$ producer response rate. The values reported throughout are representative of those who answered each survey question. A total of 217 producers from Wisconsin who raised calves responded to the survey. Further analysis was limited to respondents who performed at least some of the on-farm disbudding themselves $(87 \% ; 188 / 217)$ rather than those who outsourced disbudding to their veterinarian or veterinary technician $(13 \% ; 29 / 217)$. Of these 188 retained respondents, the mean $( \pm \mathrm{SD})$ number of heifer calves being fed milk at the time of survey response was $110 \pm 415$, and the average number of milking cows $( \pm \mathrm{SD})$ for survey respondents was $487 \pm 785$. Five $(3 \%)$ reported solely rearing calves, whereas the rest reported also milking cows. Sixty-eight percent of respondents who answered questions about their sex (n $=180$ ) were women, and $32 \%$ were men. The majority of respondents were between 35 and 54 yr old (42\%), with $34 \%$ of respondents between 18 and 34 yr old and $20 \%$ aged $55 \mathrm{yr}$ or older. The majority of respondents were owners or co-owners of their farm $(61 \%)$, followed by managers $(29 \%)$ and calf-care staff $(10 \%)$. The highest level of education completed was a 2- or 4-yr degree (53\%), followed by college or trade school (18\%), high school (13\%), and graduate degree (10\%).

With respect to respondents' calf-care management practices, the target age for calves to be completely weaned off of milk was most commonly reported as 8 to 9 wk old (57\%), followed by 6 to 7 wk old (24\%), and $10 \mathrm{wk}$ old or greater (12\%); 14 respondents (7\%) reported weaning by methods other than age. For the majority of time that their calves were on milk, re- 
spondents indicated that calves were most commonly housed individually (81\%), as opposed to other housing strategies (19\%) such as in groups, pairs, or a combination of housing styles.

Most respondents reported that 7 or more quarts of milk per day were fed to 4 -wk-old calves (58\%), whereas $40 \%$ reported less than 7 quarts per day; the question was phrased in terms of quarts, which is the most familiar unit of measure for this survey audience, and 4-wk-old calves were specified to capture the peak milk allowance. Forty-nine percent of respondents reported being somewhat satisfied by the growth performance and health of their milk-fed heifer calves, with $35 \%$ being extremely satisfied and $15 \%$ being less than satisfied (neither satisfied nor dissatisfied, somewhat dissatisfied, or extremely dissatisfied).

Respondents $(\mathrm{n}=173)$ answered questions on what resources they consult when evaluating new farming practices or technologies, with the ability to select any applicable resources. The most frequently selected options were veterinarians $(86 \%)$, other dairy producers or calf raisers $(82 \%)$, print publications $(76 \%)$, online sources $(73 \%)$, and nutritionists (72\%). Thirty-nine percent of respondents reported that they consult an extension educator for information regarding new farming practices or technologies. Of the respondents who answered questions regarding UW-Madison extension education $(\mathrm{n}=185), 55 \%$ indicated that they would be interested in receiving further information, resources, or training on dairy calf care from UW-Madison extension. Specifically, participants $(\mathrm{n}=94)$ indicated that they would be interested in learning more about disbudding and dehorning procedures (66\%), pain-control medications for disbudding or dehorning (65\%), and pair or group housing for preweaned calves (78\%). Respondents had the opportunity to select more than one of these options, as well as indicate any other topics that they were interested in through text responses.

\section{Disbudding or Dehorning Practices}

The disbudding or dehorning method was categorized based upon the most commonly used method if more than 1 method was selected by the respondent. Fifty-four percent of respondents used only 1 method of disbudding or dehorning, whereas $46 \%$ of respondents used a combination of methods. Most respondents (61\%) used caustic paste as their primary method of disbudding, followed by a hot-iron burner $(32 \%)$, and fewer reported use of surgical methods (gouge, scoop, or wire saw; $6 \%$ ).

Respondents were also asked about procedures to prepare for or follow-up after disbudding or dehorning. Of the 187 respondents who answered this question, 126
(67\%) clipped the hair of the calves before disbudding or dehorning. For caustic paste $(\mathrm{n}=114)$, a minority of respondents reported putting duct tape over the paste after application (36\%) or removing the paste a few hours after application (6\%). Of the respondents, $74 \%$ who used caustic paste, $30 \%$ of respondents who used a hot iron (18/61), and $55 \%$ of those who used surgical methods (6/11) had to repeat their disbudding or dehorning procedures at least occasionally due to a failed first attempt.

The most common indicated age for calves to be disbudded with caustic paste was on their first day of life (53\%), followed by $1 \mathrm{~d}$ to $1 \mathrm{wk}$ old $(38 \%)$, and between 1 and 4 wk of age (9\%). For respondents who used a hot iron $(\mathrm{n}=61)$, calves were most commonly disbudded at 4 to 8 wk of age (41\%), followed by 1 to 4 wk of age (33\%), greater than 8 wk of age (16\%), and $1 \mathrm{~d}$ to 1 wk old (8\%). Dehorning using surgical methods (gouge, scoop, or wire saw; $\mathrm{n}=11$ ) was most commonly reported at 8 wk of age or older (73\%). Surprisingly, some respondents reported using surgical methods to disbud calves younger than 4 wk old $(18 \%)$ or between 4 to 8 wk of age $(9 \%)$.

Fifty-five percent of respondents who answered questions related to veterinary involvement $(\mathrm{n}=185)$ indicated that their veterinarian was involved in developing their disbudding or dehorning protocol. Eighty-seven percent of respondents had regularly scheduled visits with their veterinarian (i.e., herd health).

\section{Pain Management for Disbudding or Dehorning}

Overall, $43 \%$ of respondents reported using some form of pain control for disbudding or dehorning, whereas $50 \%$ did not use any medications, and $4 \%$ were not aware of the medications that were used for disbudding or dehorning on their farm. Specifically, of the respondents who used pain control $(\mathrm{n}=81)$, an NSAID was reported to be used by $80 \%$ of respondents, followed by a local nerve block (49\%). A sedative or tranquilizer (typically xylazine) was also used by $12 \%$ of respondents. Lidocaine was the only option presented as a local anesthetic on this survey. Ten percent of respondents using lidocaine indicated that they use it buffered with sodium bicarbonate. Twenty-five percent of respondents used unbuffered lidocaine, and $65 \%$ of respondents who used lidocaine were unsure if it was buffered with sodium bicarbonate. The most common NSAID used by respondents was meloxicam (72\%), followed by flunixin meglumine (38\%), and ketoprofen $(2 \%)$. Nine percent of respondents used both meloxicam and flunixin meglumine. Forty producers $(62 \%)$ used only meloxicam, whereas $26 \%$ of respondents used only flunixin meglumine. One producer indicated that 
they exclusively used Aspirin. Most respondents indicated that their calves received only a single dose of an NSAID (74\%).

When examining drugs used in combination as well as individually, respondents $(\mathrm{n}=85)$ indicated the following: $47 \%$ of respondents used only an NSAID, $26 \%$ of respondents used an NSAID in combination with a local nerve block, $15 \%$ of respondents used only a local nerve block, $5 \%$ of respondents used only a sedative or tranquilizer, $4 \%$ of respondents used a local nerve block combined with a sedative, $2 \%$ of respondents used a combination of all 3 medications, and 1 producer used a sedative along with an NSAID.

For medications used, separated by disbudding or dehorning method, $39 \%$ of caustic-paste users, $51 \%$ of hot-iron users, and $45 \%$ of respondents using surgical methods used pain-control medications when disbudding or dehorning their calves. Lidocaine was used by $17 \%$ of respondents using caustic paste, with $30 \%$ of those using a hot iron and $18 \%$ of those using surgical methods. Nonsteroidal anti-inflammatory drugs were used by $33 \%$ of respondents disbudding with caustic paste, with $21 \%$ of those who disbud with a hot iron, and $36 \%$ of those who dehorn by surgical methods.

Respondents indicated that they had discussed paincontrol medications with their veterinarian in the last 6 mo (32\%), 6 mo to 1 yr ago (16\%), 1 to 3 yr ago (19\%), and more than $3 \mathrm{yr}$ ago $(7 \%)$. Twenty-two percent of respondents had never discussed pain-control medications with their veterinarian.

\section{Producer Justification for Using Pain Management}

Respondents were asked to select reasons why they chose to use or not use a medication for disbudding or dehorning. This question was answered by $98 \%(64 / 65)$ of NSAID users. The most commonly selected reasons for use of NSAID were importance for managing pain $(89 \%)$, availability $(64 \%)$, and reasonable cost $(44 \%)$. One hundred and fourteen respondents answered questions related to why they choose not to use an NSAID. The most commonly selected reasons for not using an NSAID were that it was not necessary for handling calves $(31 \%)$, the respondent was not familiar with the drug $(24 \%)$, or that it was not necessary for managing pain $(23 \%)$.

The most commonly selected reasons for using a local nerve block were importance for managing pain (92.5\%), ease of handling calves (70\%), and safer handling (58\%). One hundred and thirty-six respondents answered questions related to why they choose not to use a local nerve block. Respondents most commonly indicated that a local nerve block was not necessary for managing pain $(37 \%)$, they were not familiar with the drug or procedure $(30 \%)$, or it took too much time to administer (24\%).

Respondents reporting use of a sedative $(\mathrm{n}=10)$ most commonly stated this was used because it made handling calves easier (90\%) and safer (90\%). One hundred and sixty-seven respondents answered questions related to why they do not use a sedative for disbudding or dehorning. Respondents reported that sedatives were not necessary for handling calves (46\%), they were not familiar with the drug $(32 \%)$, it took too much time to administer $(19 \%)$, or was not necessary for managing pain (17\%).

A logistic regression model was built to determine factors associated with the use of pain control. Pain control was defined as use of either a local anesthetic or an NSAID, or both; use of sedation alone was not considered a pain-control strategy. Herd size (categorical), average pain score in response to the 5 disbudding and dehorning scenarios (continuous), veterinary involvement (yes or no), and disbudding method (categorical) were offered to a multivariable model. In the final model, veterinarian involvement in creating a disbudding protocol was the sole significant variable. Respondents who had veterinary involvement in the disbudding protocol had increased odds of using paincontrol medication (odd ratio $=4.22 ; 95 \% \mathrm{CI}=2.24$, 7.94; $P<0.001)$ when compared with respondents who did not have a veterinarian involved in the protocol.

\section{Pain Perception Toward Disbudding or Dehorning}

The respondents' perceptions of calf pain when disbudding or dehorning using 5 methods (caustic paste on d 1 of life, caustic paste on d 10, hot iron on d 10 , hot iron at $7 \mathrm{wk}$, gouge and hot iron at $5 \mathrm{mo}$ ) were evaluated, along with the respondents' primary disbudding or dehorning methods. Respondents were told to assess calf pain to the application of these 5 methods without the use of pain control. On average, respondents viewed these 5 procedures as follows, from most painful to least painful: gouge and hot iron at 5 mo, hot iron at $7 \mathrm{wk}$, hot iron at $10 \mathrm{~d}$, caustic paste at $10 \mathrm{~d}$, caustic paste at $1 \mathrm{~d}$ (Figure 1). Respondents who used caustic paste as their primary disbudding method perceived the application of caustic paste on 1-d-old calves without pain mitigation to be less painful than respondents who used hot iron as their primary disbudding method $(P=0.02$; Figure 2a). However, when examining other comparisons of disbudding and dehorning methods, no significant differences in pain perception were found between other comparisons. Differences were found between all 2-way comparisons of the following 5 disbudding and dehorning scenarios performed without pain mitigation: caustic paste ap- 
plied on d 1, caustic paste applied on d 10, a hot iron used on d 10, a hot iron used at wk 7 , gouge and hot iron used at 5 mo $(P \leq 0.013$ for all comparisons; average pain score distributions represented in Figure 1).

\section{Compliance with FARM Expectations}

As of January 1, 2020, version 4.0 of the FARM program standards included that disbudding must be conducted before 8 wk of age, some form of pain control must be used, and a veterinarian must be involved in the creation of the farm's disbudding protocol. It is important to note that this survey was conducted in the fall of 2019, before implementation of these new standards. When considering producers who were in compliance with the FARM requirement for disbudding age, $90 \%$ of respondents disbudded before calves reached $8 \mathrm{wk}$ of age. Compliance with this requirement varied by primary disbudding or dehorning method. All respondents primarily using caustic paste $(\mathrm{n}=114)$ performed disbudding before 8 wk of age. Of respondents using hot iron $(\mathrm{n}=61), 82 \%$ disbudded before $8 \mathrm{wk}$ of age, and $27 \%$ using surgical methods (gouge, scoop or wire saw; $\mathrm{n}=11$ ) did so before 8 wk of age. Compliance with use of pain-control medication for disbudding and dehorning was achieved by $43 \%$ of respondents. Fiftyfive percent of respondents who answered questions

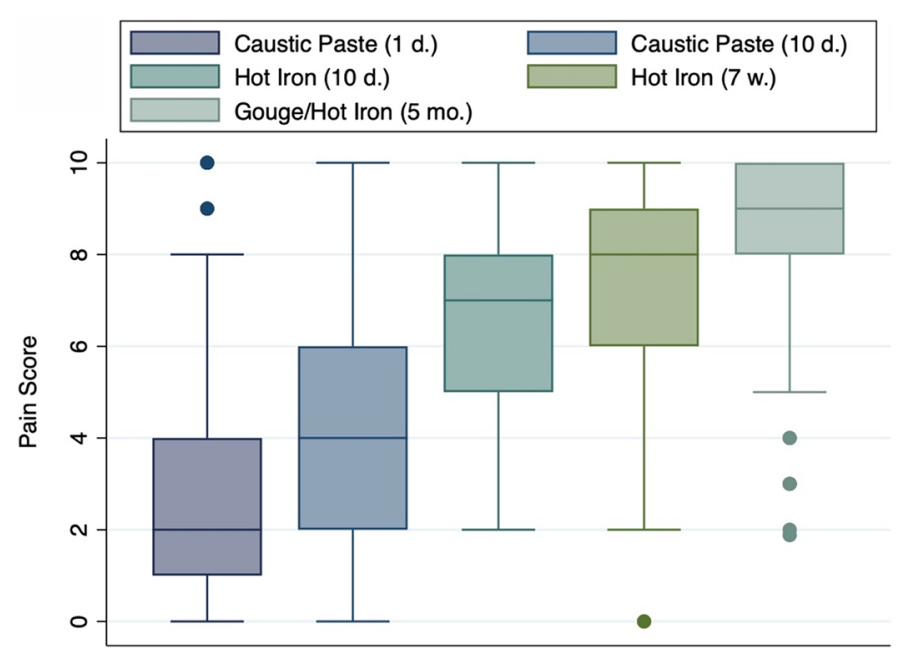

Figure 1. Distributions of respondents' pain perception scores for the following 5 disbudding and dehorning scenarios: caustic paste d 1 of life $(\mathrm{n}=166)$, caustic paste on d $10(\mathrm{n}=175)$, hot iron on $d 10(\mathrm{n}$ $=176)$, hot iron at $7 \mathrm{wk}(\mathrm{n}=177)$, gouge + hot iron at $5 \mathrm{mo}(\mathrm{n}=$ 173). Boxes represent the interquartile range (IQR; Q1-Q3), with the median of each distribution represented by the darker line within the box. The top and bottom of the box represent the 75th and 25th percentiles, with the vertical whiskers showing the minimum (Q1 $-1.5 \times$ $\mathrm{IQR})$ and maximum $(\mathrm{Q} 3-1.5 \times \mathrm{IQR})$, and dots reflecting outlier values. Kolmogrov-Smirnov tests for equality distribution were performed between all possible 2-way comparisons. Significant differences were found between all 2-way comparisons ( $P \leq 0.013$ for all comparisons). about veterinary involvement $(\mathrm{n}=185)$ indicated that their veterinarian was involved in developing their current disbudding or dehorning protocol, indicating that they were in compliance with this FARM requirement. When these data were combined, $32 \%$ of respondents were compliant with all 3 expectations for disbudding age, use of a pain-control medication (local anesthetic or NSAID), and involvement of the veterinarian.

Respondents who had a target weaning age of 6 to 7 wk and those with a target of 8 to 9 wk had lower odds of complying with FARM requirements when compared with respondents with a target weaning age of $\geq 10 \mathrm{wk}$ (Table 1). Herd size was seen to be a confounder of this relationship due to a difference of $>20 \%$ in the coefficients of weaning age when it was removed from the model; therefore, it was retained in the final model.

\section{Changes to Disbudding and Dehorning Protocols}

A total of 137 (73\%) respondents indicated that they had changed their pain-control medications, disbudding method, or disbudding age in the past $10 \mathrm{yr}$. Univariable logistic regression analysis showed potential associations between changing disbudding practices in the last $10 \mathrm{yr}$ and respondent age (categorical: 18-34, $35-54, \geq 55$ ), education (categorical: high school, college or trade school, 2- or 4-yr degree, graduate degree), whether or not producers have regularly scheduled visits with their veterinarian (yes or no), target weaning age (categorical: $6-7 \mathrm{wk}, 8-9 \mathrm{wk}, \geq 10 \mathrm{wk}$, other), calf housing (individual vs. other social groupings), farmer satisfaction with calf health (categorical: less than satisfied, somewhat satisfied, extremely satisfied), repeat disbudding (yes or no), and herd size (categorical: $0-12,13-30,31-60,>60)$. Multivariate analysis showed that respondents between the ages of 18 to $34 \mathrm{yr}$ had greater odds of having made changes to their disbudding protocol compared with those aged 35 to $54 \mathrm{yr}$ (Table 2). Compared with respondents with the largest farms ( $>60$ calves), respondents with $\leq 60$ calves had lower odds of having made changes to their disbudding protocol (Table 2).

\section{Influences on Change to Disbudding and Dehorning Practices}

For those reporting changing their disbudding or dehorning methods (i.e., type of disbudding or age at disbudding) in the past $10 \mathrm{yr}(\mathrm{n}=116)$, respondents were asked to select who or what influenced their decision, and multiple options could be selected. The most commonly selected influences were veterinarian $(62 \%)$, followed by farm staff or family members $(31 \%)$, other farmers $(23 \%)$, and public perception or consumer 

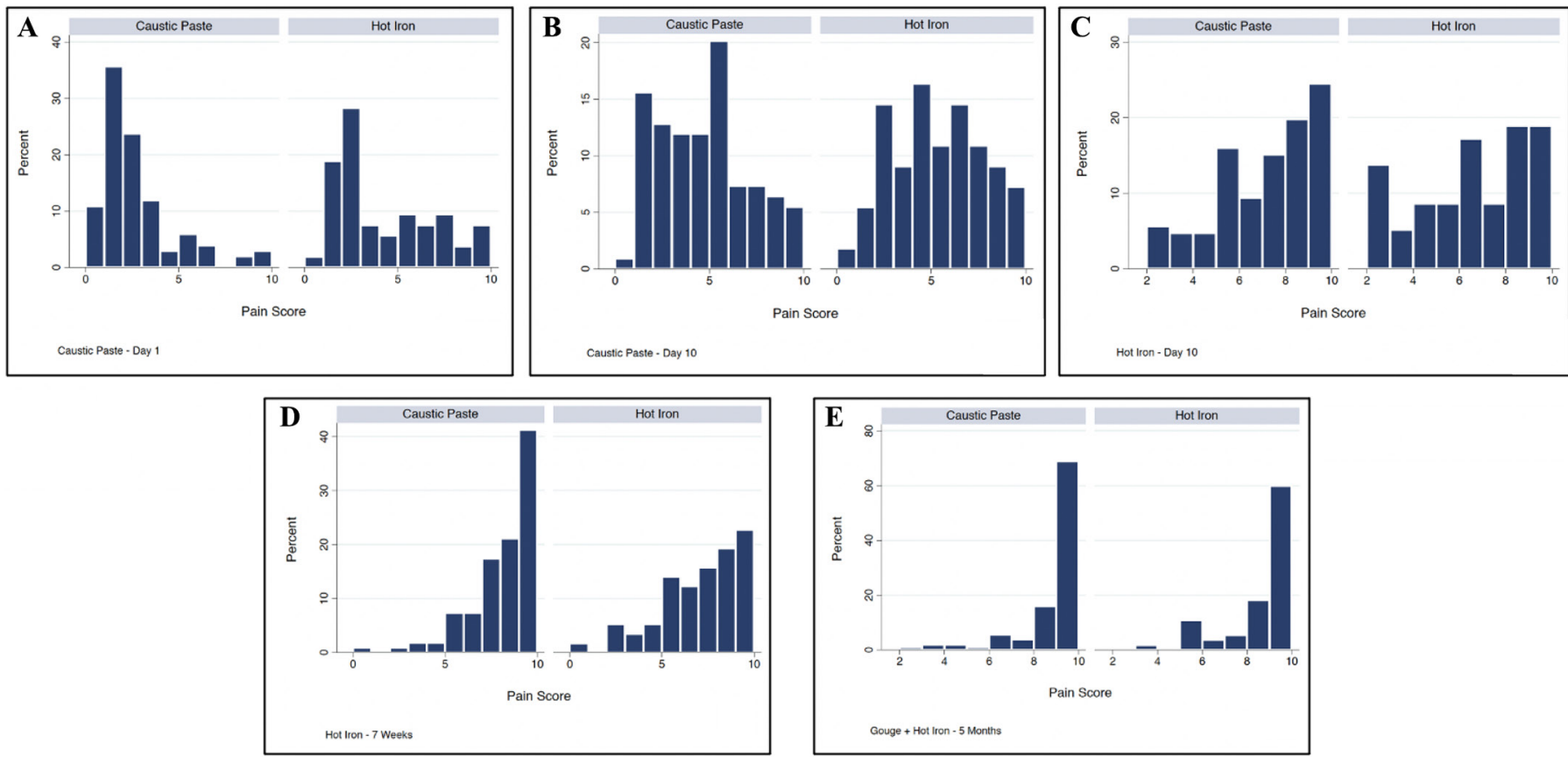

Figure 2. Distribution of respondents' pain perception scores associated with 5 different disbudding and dehorning scenarios, all without pain control, given as follows: caustic paste on d 1 of life (A), caustic paste on d 10 (B), hot iron on d 10 (C), hot iron at 7 wk (D), hot iron + gouge at 5 mo (E), by type of primary disbudding method used on the respondent's farm (caustic paste or hot iron). Differences between respondent groups (caustic paste vs. hot iron) were assessed using the Komelgrov-Smirnov test for equality of distribution. The only scenario with a different equality of distribution was in scenario A, where caustic paste was used on 1-d-old calves $(P=0.020)$, in which caustic paste users rated the procedure as less painful than hot-iron users.

demand (23\%). Farm advisors (e.g., extension agents, nutritionists) were reported to influence disbudding practices for only $18 \%$ of respondents. Similarly, for respondents who indicated that they had changed their use of pain-control medication in the last $10 \mathrm{yr}$ $(\mathrm{n}=96)$, the most commonly selected influences were veterinarian $(79 \%)$, public perception or consumer demand $(29 \%)$, and farm staff or family members $(19 \%)$. Extension educators and other farm advisors (e.g., nu-

Table 1. Results from multivariable logistic regression model evaluating the association between herd size, target weaning age, and the odds of complying with Farmers Assuring Responsible Management (FARM) version 4.0 requirements among 187 respondents

\begin{tabular}{lccc}
\hline Variable & $\begin{array}{c}\text { Odds } \\
\text { ratio }\end{array}$ & $95 \%$ CI & $P$-value \\
\hline Herd size (number of calves) & Referent & & \\
$0-12$ & 1.11 & $0.43-2.90$ & 0.83 \\
12-30 & 1.09 & $0.41-2.84$ & 0.87 \\
$30-60$ & 0.71 & $0.28-1.80$ & 0.47 \\
$\quad>60$ & & & \\
Target weaning age (wk) & 0.112 & $0.01-0.97$ & 0.046 \\
$\quad$-7 & 0.093 & $0.01-0.75$ & 0.026 \\
$8-9$ & Referent & & \\
$\geq 10$ & 0.188 & $0.017-2.11$ & 0.176 \\
Method other than calf age & 19.47 & & \\
Constant & &
\end{tabular}

tritionist) were reported to influence use of pain-control medication for only $16 \%$ of respondents.

\section{Use of Polled Genetics}

A total of 84 respondents indicated that they currently use some amount of polled genetics in their herd. Education level (categorical: high school, college or trade school, 2- or 4-yr degree, graduate degree),

Table 2. Results from multivariable logistic regression model evaluating the association between herd size, respondent age, and the odds of having changed the farm's disbudding protocol, disbudding method, or pain-control medications in the last $10 \mathrm{yr}$ among 181 respondents

\begin{tabular}{lccc}
\hline Variable & $\begin{array}{c}\text { Odds } \\
\text { ratio }\end{array}$ & $95 \% \mathrm{CI}$ & $P$-value \\
\hline $\begin{array}{l}\text { Herd size (number of calves) } \\
0-12\end{array}$ & 0.041 & $0.00-0.33$ & 0.003 \\
$12-30$ & 0.046 & $0.01-0.38$ & 0.004 \\
$30-60$ & 0.066 & $0.01-0.54$ & 0.011 \\
$\quad>60$ & Referent & & \\
$\begin{array}{l}\text { Respondent age (yr) } \\
\quad 18-34\end{array}$ & 3.49 & $1.39-8.73$ & 0.008 \\
$35-54$ & Referent & & \\
$\geq 55$ & 1.64 & $0.62-4.33$ & 0.315 \\
Constant & 28.52 & & \\
\hline & & & \\
\end{tabular}


Table 3. Results from multivariate logistic regression model evaluating association between herd size, weaning age, and the odds of using any polled genetics in the dairy herd among 183 respondents

\begin{tabular}{|c|c|c|c|c|}
\hline Variable & & $\begin{array}{l}\text { Odds } \\
\text { ratio }\end{array}$ & $95 \% \mathrm{CI}$ & $P$-value \\
\hline Herd size & For every 1 calf increase & 1.00 & $0.99-1.00$ & 0.207 \\
\hline \multirow[t]{4}{*}{ Target weaning age } & $6-7 \mathrm{wk}$ & 0.21 & $0.06-0.70$ & 0.01 \\
\hline & $8-9 \mathrm{wk}$ & 0.33 & $0.11-0.996$ & 0.049 \\
\hline & $\geq 10 \mathrm{wk}$ & Referent & & \\
\hline & $\bar{M}$ ethod other than calf age & 0.20 & $0.04-0.88$ & 0.034 \\
\hline Constant & & 3.04 & & \\
\hline
\end{tabular}

herd size (continuous), regularly scheduled visits with their veterinarian (yes or no), and target weaning age (categorical: 6-7 wk, 8-9 wk, $\geq 10 \mathrm{wk}$, other) were significant in univariable analysis and were offered to the multivariable logistic regression model. Respondents who had a target weaning age of 6 to 7 wk, 8 to 9 wk, and those who wean by methods other than age had lower odds of using polled genetics compared with those who have a target weaning age of $\geq 10 \mathrm{wk}$ (Table $3)$. Herd size was identified as a confounder of this relationship due to a difference of $>20 \%$ in the coefficients of weaning age when it was removed from the model; therefore, it was retained in the final model.

\section{DISCUSSION}

The objectives of this study were to identify current disbudding and dehorning practices of dairy farmers in Wisconsin to better target extension programming for the adoption of pain control when disbudding calves. Caustic paste was the most common method of disbudding or dehorning used by respondents to this survey. Most respondents who disbudded using this method did so within the first week of the calf's life. Paincontrol medications in the form of a local anesthetic or an NSAID were used by $43 \%$ of respondents when disbudding or dehorning calves, and those whose veterinarians were involved in creating their disbudding or dehorning protocol had greater odds of using pain control. Veterinarians were a prominent source of influence on respondents, along with farm staff or family members, other farmers, and public perception or consumer demand. Younger respondents and respondents with larger herds were associated with increased odds of having made changes to their disbudding or dehorning protocol in the last decade. An association was found between respondents with progressive weaning strategies (such as weaning with a target age of $10 \mathrm{wk}$ old or greater) and the inclusion of some polled genetics in their dairy herd, as well as compliance with FARM v. 4.0 requirements.

\section{Respondent Demographics}

Most of the respondents were female and between 35 and $54 \mathrm{yr}$ of age. According to the 2017 Census of Agriculture (USDA, 2019), the average age for US dairy producers was $50 \mathrm{yr}$ old, with most $(70 \%)$ being male, indicating that the population of this survey may not be representative of the larger dairy-producer population in the United States. The average number of milking cows for survey respondents was $487 \pm 785$ (mean $\pm \mathrm{SD}$ ). This is considerably larger than the average herd size for Wisconsin dairy farms in 2014, which was 110 milking cows (USDA, 2018). These respondent demographics may reflect those who are more likely to engage with extension education and the industry networks who distributed the survey (e.g., veterinarians).

\section{Disbudding and Dehorning Methods}

An average of $61 \%$ of producers who responded to this study disbudded calves using caustic paste. Previous studies have shown that the majority of American producers use a hot-iron burner as their primary disbudding method rather than caustic paste. The National Animal Health Monitoring System survey identified that in 2014, caustic paste was used on $32.5 \%$ of disbudded calves in the United States (USDA, 2018). The results of this survey suggested that, in Wisconsin, the use of caustic paste as a primary disbudding method may be more popular than previous studies have shown. In Canada, however, caustic paste is seen to be less popular, with the majority of producers primarily using a hot-iron burner to disbud their calves (Vasseur et al., 2010; Winder et al., 2016, 2018a). A slight increase in the number of Ontario producers using caustic paste (13\% in 2016 compared with $9 \%$ in 2007) may suggest that although less popular than a hot iron, use of caustic paste for disbudding may be increasing (Winder et al., 2016; Misch et al., 2007)

The large proportion of survey respondents that disbudded using caustic paste could be a result of their 
perceptions regarding the pain associated with caustic paste. For the respondents using caustic paste, a larger proportion disbudded without the use of pain mitigation. Respondents to this survey viewed caustic paste disbudding as less painful than hot-iron disbudding or amputation methods (i.e., gouge combined with hot iron). Although evidence exists to support the fact that amputation dehorning is more painful than hot iron or caustic paste disbudding (Stafford and Mellor, 2011; Stock et al., 2013), there is very little research that accurately compares disbudding methods directly. The lack of research in this area makes it difficult to draw accurate comparisons between the pain associated with caustic paste and hot-iron disbudding. It may be the case that the respondents to this survey use caustic paste as their primary method of disbudding because they perceive it to be a less painful form of disbudding. This belief may also explain why a large proportion of respondents who primarily disbud with caustic paste do so without pain mitigation.

\section{Pain-Control Practices}

Just $43 \%$ of our respondents reported using pain mitigation for disbudding and dehorning. In addition, despite the use of both an NSAID and a local anesthetic being identified as best practice by veterinary organizations (CVMA, 2016; AABP, 2019), only $12 \%$ of respondents indicated that they use both medications. The results of this survey suggest an increase in use of pain management from what was previously found in Wisconsin (18\% using a local anesthetic; Hoe and Ruegg, 2006) and more widely across the United States (28\% using analgesics or anesthetics; USDA, 2018; less than $20 \%$ using local or systemic analgesia the majority of the time; Johnstone, et al., 2021). However, compared with Canadian producers, the number of respondents using pain management remains low. In Ontario, $62 \%$ of producers choose to use local anesthetic and $24 \%$ chose to use an NSAID when performing disbudding or dehorning on their own farm (Winder et al., 2016). The greater number of Canadian producers disbudding and dehorning with pain management may be a result of pain-control recommendations and requirements that have been in place for substantially longer (since 2009; NFACC, 2009) than the requirements for American dairy farmers. The larger proportion of Canadian producers now using pain management compared with previous studies suggests that the newly implemented FARM standards may improve disbudding practices for American producers. A future follow-up study could test this hypothesis.
Survey respondents' motivations for and barriers against using pain control for disbudding are similar to what was previously found with producers in Ontario (Winder et al., 2016). Our study revealed that producers' views of pain control are an important factor in understanding why, or why not, respondents chose to use pain mitigation for disbudding practices. As with the Winder et al. (2016) study, producer opinions on the necessity of pain control for handling and managing pain, their familiarity with the drugs, and their perception of the time associated with this procedure greatly influenced their choices to use, or not use, these medications. Theories of behavior have shown that an individual's attitudes and beliefs largely affect their willingness to perform certain behaviors (Ajzen, 1991), as well as change their current patterns of behavior (Prochaska and Di Clemente, 1982). Producers' personal beliefs and opinions have been seen to influence their decisions to implement disease prevention programs on their farms (Sorge et al., 2010; Brennan et al., 2016; Richens et al., 2018) and their decisions to keep horned or dehorned cattle (Kling-Eveillard et al., 2015; Cardoso et al., 2016). The results of this study highlighted the importance of understanding producers' perspectives to certain animal welfare practices to better target producer education.

Although requirements for dairy producers differ across North America, the results of this study showed that pain management concerns are similar for American and Canadian producers. Recognizing this allows researchers to better understand the relationship between producer motivations and their on-farm practices, which can help to guide future extension programming and producer education. By developing a better understanding of producers' opinions toward the use of pain control for disbudding and dehorning, we are able to develop extension programming that caters toward the needs of dairy farmers and encourages further education on the necessity of pain mitigation for this practice.

Survey results indicated that respondents viewed hot-iron disbudding and caustic paste disbudding to be less painful in younger calves than older calves. Some evidence suggests the opposite to be true with the potential for a long-term increase in pain sensitivity in calves disbudded soon after birth (Adock and Tucker, 2018). The study by Adock and Tucker (2018) found that calves disbudded at $3 \mathrm{~d}$ of age were more sensitive to rump pressure than calves disbudded at $35 \mathrm{~d}$ of age, suggesting that painful stimuli early in a calf's life may lead to longer term pain sensitivity. Despite the little work that has been done in the area of disbud- 
ding age and calf pain, some veterinary organizations recommend that disbudding be done at the earliest age possible (AABP, 2019; AVMA, 2021), which may encourage the perception that disbudding calves is less painful at a younger age.

\section{Changes to Disbudding or Dehorning Protocol}

Respondents in the youngest age category (18-35 yr of age) and respondents with larger herds ( $>60$ calves) were more likely to have changed either their disbudding or dehorning method, calf age when the procedure is performed, or use of medications in the last decade. A previous study by Wikman et al. (2013) that investigated Finnish dairy-producer attitudes to pain when disbudding calves identified that younger producers (under $39 \mathrm{yr}$ old) were more willing to medicate their calves than older producers. Perhaps an explanation for the increase seen in pain control use is that younger producers may be more willing to implement the use of pain-control medication on their farms. With respect to herd size, Hoe and Ruegg (2006) previously found larger herds in Wisconsin were more likely to adopt biosecurity protocols, perform mastitis control procedures, and be enrolled in a Johne's disease control program. Producers with larger herds may have the opportunity to implement better management practices, as evidenced by larger Australian herds having higher adoption of precision dairy technologies (Gargiulo et al., 2018). Farms with larger herds also have the potential for additional staff and personnel, greater access to a veterinarian, larger facilities, and increased resources (Hoe and Ruegg, 2006), which may make changes to on-farm protocols more feasible.

\section{Producer Influences}

An association was found between using pain control and having a veterinarian involved in creating an onfarm disbudding or dehorning protocol, highlighting the veterinarian's role in advocating for pain management. This is similar to findings from Ontario (Winder et al., 2016) and Kentucky (Russell and Bewley, 2013). Misch et al. (2007) suggested that when veterinarians are involved in on-farm dehorning decisions, they are the main source of influence on a producer's decision to use medication for the procedure.

In addition to veterinarians, public perception and consumer demand are viewed as influential to respondents to this survey. Wolf et al. (2016) found that consumers were viewed by farmers as being highly influential on expectations for dairy cattle welfare. Stakeholder views are important in developing sustainable agriculture systems and future legislation (Robbins et al., 2015; Cardoso et al., 2016); therefore, the influential presence of the public is not surprising within this study.

The need for further extension efforts within this population is clear, with over half of the respondents to this survey indicating that they would be interested in receiving further information from UW-Madison extension. Despite few respondents reporting extension educators as influential on pain control use, the high interest in extension education is promising and demonstrates the potential that extension programming has to encourage best practices among producers.

\section{Producer Compliance}

Approximately $32 \%$ of our respondents were compliant with all 3 of the disbudding and dehorning standards from the 2020 FARM v. 4.0 program. This survey was conducted in 2019, before the FARM v. 4.0 disbudding and dehorning expectations were implemented. It may be the case that respondents who were not in compliance with FARM requirements for disbudding and dehorning at the time of the survey have since changed their practices to comply with the standards implemented on January 1, 2020. Additionally, this analysis only focused on Wisconsin respondents who performed disbudding and dehorning themselves, which eliminated potentially compliant respondents who outsourced their disbudding or dehorning to a veterinarian. Respondents who had a target weaning age of $\geq 10 \mathrm{wk}$ were more likely to be in compliance with FARM disbudding requirements. Delayed weaning age has been demonstrated to show improvements in calf energy intake and average daily gain (de Passillé et al., 2011) and may be an indicator of more progressive calf-care practices. The disbudding standards from FARM, although previously recommended by the program, have been newly elevated. It is possible that producers in this survey population who practiced more progressive weaning strategies were more willing to adopt other novel practices, such as best practices for disbudding and dehorning.

\section{Polled Genetics}

Respondents who delayed weaning were also more likely to incorporate some polled genetics into their herd. The use of polled genetics in dairy and beef farming is a controversial practice among producers due to the potential sacrifice of other beneficial traits for the polled gene (Anderson, 2009; Meyer, 2015). The US Department of Agriculture reported that in 2013, approxi- 
mately one-quarter of dairy operations in the United States used polled genetics (USDA, 2018); however, almost half of the respondents to this study indicated that they used some polled genetics in their herd. This may suggest that compared with previous estimates, the use of polled genetics on American dairy farms has increased in recent years. This also supports the idea that producers who are willing to adopt progressive weaning strategies may be more willing to incorporate other progressive calf-care practices on their farms, such as the use of polled genetics.

\section{Study Implications}

The results of this study highlighted the effect of veterinarians as an influential source on dairy producers, as well as the importance of producers' opinions in determining their on-farm practices. Justification of producers' choices to use, or not use, pain-control medication for disbudding and dehorning was heavily reliant on the producers' perception of the necessity of these drugs for horn removal procedures. Developing a better understanding of the perspectives of American dairy farmers allows extension agents and veterinarians to better tailor their educational resources for producers, creating more effective and impactful educational programming. With targeted extension programming, veterinarians, extension agents, and other industry stakeholders can encourage the use of pain control for disbudding and dehorning by highlighting the necessity of pain mitigation for these procedures and creating a narrative around pain control use that is better tailored to individual producers.

\section{Survey Limitations}

There are several limitations to consider when interpreting the results of this study. Convenience sampling was used to target the respondents to this survey, which was done to ensure that the survey population was reflective of producers who were interested in extension programming and whom extension programming would reach. However, this means that our sample may not have necessarily reflected the opinions of the larger Wisconsin dairy-producer population. Surveys were distributed directly to producers through study team members and industry contacts, which further increased the risk of bias in this study. This distribution strategy had the potential to overrepresent participants who had positive relationships with industry professionals, such as extension educators or veterinarians, and may not have allowed all members of the population to be accurately represented in this sample. Producers who look to interact with extension agents and industry professionals may be more inclined to follow changing industry standards, which could indicate that the use of practices such as pain control or polled genetics were overrepresented in this study. The sampling strategy potentially reduced the external validity of these survey results due to the inability to extrapolate data to the larger population of dairy farmers within the state. However, even among this seemingly progressive population, many opportunities for improved education were identified, particularly in terms of pain control use for disbudding.

An additional limitation to consider is that respondents to this survey were sampled ahead of the new FARM standards becoming mandatory in 2020. This approach only allowed for the representation of the compliance of respondents to practices that were recommended, but not expected (i.e., pain-control medications and veterinary involvement in the disbudding protocol) or which had a longer timescale for involvement (i.e., disbudding by 8 wk of age) at the time of sampling. Therefore, responses to this survey may not accurately represent producers' willingness to adopt practices in light of the since-increased expectations. Future research could examine whether compliance improves after the latest FARM expectations have been in place for a few years, with additional targeted extension programming, or a combination of these factors.

\section{CONCLUSIONS}

Caustic paste was identified as the most commonly used disbudding method by respondents, followed by a hot-iron burner. The findings of this research highlighted an increase in the adoption of pain-control medication by Wisconsin dairy producers when disbudding or dehorning their calves, as well as the use of polled genetics, compared with previous research. The use of both practices is greater in this sample relative to previous work; however, the results of this survey indicated that the number of producers who disbud without pain management is still high. This presents substantial challenges in light of the recently updated FARM requirements and highlights the need for targeted extension programming to encourage the adoption of best disbudding practices by producers. Veterinarians were seen to be the greatest source of influence on respondent adoption of pain-control practices. This demonstrated the opportunity that veterinarians have in increasing potential adoption of best practices by producers through education and awareness. 


\section{ACKNOWLEDGMENTS}

This project was funded by an Animal Well-Being Subgroup Mini Grant through the University of Wisconsin (UW)-Madison Division of Extension (Madison, WI). Gifts in kind in the form of raffle drawing prizes for survey participants were provided by the Coburn Company Inc. (Whitewater, WI), Calf-Tel (Hampel Corp., Germantown, WI), and Vita Plus Corporation (Madison, WI). We are grateful to Matthew Akins (Department of Dairy and Animal Sciences, UW-Madison), Theresa Ollivett (School of Veterinary Medicine, UWMadison), Tina Kohlman, Heather Schlesser, Becky Schlev, Sandra Stuttgen, and Jim Versweyveld (all affiliated with UW-Madison Division of Extension at the time of the survey) for their assistance with creating and disseminating the survey. The authors declare no conflicts of interest.

\section{REFERENCES}

AABP (American Association of Bovine Practitioners). 2019. American Association of Bovine Practitioners dehorning guidelines. AABP.

Adock, S. J. J., and C. B. Tucker. 2018. The effect of disbudding age on healing and pain sensitivity in dairy calves. J. Dairy Sci. 101:10361-10373. https://doi.org/10.3168/jds.2018-14987.

Ajzen, I. 1991. The theory of planned behaviour. Organ. Behav. Hum. Decis. Process 50:179-211. https://doi.org/10.1016/0749 -5978(91)90020-T.

American Veterinary Medical Association (AVMA). 2014. Literature review on the welfare implications of the dehorning and disbudding of cattle. Accessed Jan. 18, 2021. http://cmapspublic3.ihmo .us/rid=1QL1WD46J-1PJVPY6-FR/dehorning_cattle_bgnd.pdf.

American Veterinary Medical Association (AVMA). 2021. Castration and dehorning of cattle. Accessed Jan. 18, 2021. https:/ /www.avma.org/resources-tools/avma-policies/castration-and -dehorning-cattle.

Anderson, N. 2009. Dehorning of calves factsheet. Accessed Nov. 28, 2019. http://www.omafra.gov.on.ca/english/livestock/dairy/facts/ 09-003.htm\#dehorn.

Bates, A. J., R. A. Laven, F. Chapple, and D. S. Weeks. 2016. The effect of different combinations of local anaesthesia, sedative and non-steroidal anti-inflammatory drugs on daily growth rates of dairy calves after disbudding. N. Z. Vet. J. 64:282-287. https://doi .org/10.1080/00480169.2016.1196626.

Brennan, M., N. Wright, W. Wapenaar, S. Jarratt, P. Hobson-West, I. Richens, J. Kaler, H. Buchanan, J. Huxley, and H. O'Connor. 2016. Exploring attitudes and beliefs towards implementing cattle disease prevention and control measures: A qualitative study with dairy farmers in Great Britain. Animals (Basel) 6:61. https://doi .org/10.3390/ani6100061.

Canadian Veterinary Medical Association (CVMA). 2016. Disbudding and dehorning of cattle - position statement. Accessed Nov. 28,2019 . https://www.canadianveterinarians.net/documents/ disbudding-and-dehorning-of-cattle.

Caray, D., A. de Boyer des Roches, S. Frouja, S. Andanson, and I. Veissier. 2015. Hot-iron disbudding: Stress responses and behaviour of 1 - and 4-week-old calves receiving anti-inflammatory analgesia without or with sedation using xylazine. Livest. Sci. 179:2228. https://doi.org/10.1016/j.livsci.2015.05.013.

Cardoso, C. S., M. J. Hotzel, D. M. Weary, J. A. Robbins, and M. A. G. von Keyserlingk. 2016. Imagining the ideal dairy farm. J. Dairy Sci. 99:1663-1671. https://doi.org/10.3168/jds.2015-9925.
Cozzi, G., F. Gottardo, M. Brscic, B. Contiero, N. Irrgang, U. Knierim, O. Pentelescu, J. J. Windig, L. Mirabito, F. Kling Eveillard, A. C. Dockes, I. Veissier, A. Velarde, C. Fuentes, A. Dalmau, and C. Winckler. 2015. Dehorning of cattle in the EU Member States: A quantitative survey of the current practices. Livest. Sci. 179:4-11. https://doi.org/10.1016/j.livsci.2015.05.011.

de Passillé, A. M., T. F. Borderas, and J. Rushen. 2011. Weaning age of calves fed a high milk allowance by automated feeders: Effects on feed, water, and energy intake, behavioural signs of hunger, and weight gains. J. Dairy Sci. 94:1401-1408. https://doi.org/10.3168/ jds.2010-3441.

Farmers Assuring Responsible Management (FARM). 2020. Animal care version 4.0 requirements \& corrective actions. Accessed on Feb. 24, 2021. https://nationaldairyfarm.com/wp-content/ uploads/2020/09/FARM_Animal-Care-4-Manual_Layout_FINAL _091520_SinglePages.pdf

Fulwider, W. K., T. Grandin, B. E. Rollin, T. E. Engle, N. L. Dalsted, and W. D. Lamm. 2008. Survey of dairy management practices on one hundred thirteen North Central and Northeastern United States dairies. J. Dairy Sci. 91:1686-1692. https://doi.org/10 $.3168 /$ jds.2007-0631.

Gargiulo, J. I., C. R. Eastwood, S. C. Garcia, and N. A. Lyons. 2018. Dairy farmers with larger herd sizes adopt more precision dairy technologies. J. Dairy Sci. 101:5466-5473. https://doi.org/10 $.3168 /$ jds.2017-13324.

Hambleton, S. Y. N., and T. J. Gibson. 2017. Study investigating the attitudes and opinions of cattle farmers and veterinarians in the UK on the use of non-steroidal anti-inflammatory drugs (NSAIDs) for post-disbudding analgesia of calves. Anim. Welf. 26:322-333. https://doi.org/10.7120/09627286.26.3.323.

Hoe, F. G. H., and P. L. Ruegg. 2006. Opinions and practices of Wisconsin dairy producers about biosecurity animal well-being. J. Dairy Sci. 89:2297-2308. https://doi.org/10.3168/jds.S0022 $-0302(06) 72301-3$.

Johnstone, E. C. S., J. F. Coetzee, P. J. Pinedo, and L. EdwardsCallaway. 2021. Current attitudes of veterinarians and producers regarding the use of local and systemic analgesia in beef and dairy cattle in the United States. J. Am. Vet. Med. Assoc. 258:197-209. https://doi.org/10.2460/javma.258.2.197.

Kling-Eveillard, F., U. Knierim, N. Irrgang, F. Gottardo, R. Ricci, and A. C. Dockes. 2015. Attitudes of farmers towards cattle dehorning. J. Livest. Sci. 179:12-21. https://doi.org/10.1016/j.livsci.2015.05 .012 .

Meyer, C. Progressive Dairy. 2015. The pros and cons of polled genetics. Accessed Jun. 1, 2020. https://www.progressivedairycanada .com/topics/a-i-breeding/the-pros-and-cons-of-polled-genetics.

Misch, L. J., T. J. Duffield, S. T. Millman, and K. D. Lissemore. 2007. An investigation into the practices of dairy producers and veterinarians in dehorning dairy calves in Ontario. Can. Vet. J. 48:1249-1254.

National Dairy Farm Program. 2020. 2020 year in review. Accessed Apr. 29, 2021. https://nationaldairyfarm.com/wp-content/ uploads/2021/02/FARM_YearInReview_2020_FINAL_022421 _Web_pages.pdf

National Farm Animal Care Council (NFACC). 2009. Husbandry practices, disbudding and dehorning. Page 32 in Code of Practice for the Care and Handling of Dairy Cattle. National Farm Animal Care Council (NFACC).

Prochaska, J. O., and C. C. Di Clemente. 1982. Transtheoretical therapy: Toward a more integrative model of change. Psychotherapy (Chic.) 19:276-288. https://doi.org/10.1037/h0088437.

Reedman, C. N., T. F. Duffield, T. J. DeVries, K. D. Lissemore, N. A. Karrow, Z. Li, and C. B. Winder. 2020b. Randomized control trial assessing the efficacy of pain control strategies for caustic paste disbudding in dairy calves younger than 9 days of age. J. Dairy Sci. 103:7339-7350. https://doi.org/10.3168/jds.2019-18118.

Reedman, C. N., T. F. Duffield, T. J. DeVries, K. D. Lissemore, and C. B. Winder. 2020a. The effects of xylazine sedation in 2- to 6-wkold calves disbudded with a cautery iron. J. Dairy Sci. 103:(Suppl 1):86-87. 
Richens, I. F., J. Houdmont, W. Wapenaar, O. Shortall, J. Kaler, H. O'Connor, and M. L. Brennan. 2018. Application of multiple behaviour change models to identify determinants of farmers' biosecurity attitudes and behaviours. Prev. Vet. Med. 155:61-74. https://doi.org/10.1016/j.prevetmed.2018.04.010 https:dx.doi .org/j.prevetmed.2018.04.010.

Robbins, J. A., D. M. Weary, C. A. Schuppli, and M. A. G. von Keyserlingk. 2015. Stakeholder views on treating pain due to dehorning dairy calves. Anim. Welf. 24:399-406. https://doi.org/10.7120/ 09627286.24.4.399.

Russell, R. A., and J. M. Bewley. 2013. Characterization of Kentucky dairy producer decision-making behaviour. J. Dairy Sci. 96:47514758. https://doi.org/10.3168/jds.2012-6538.

Sargeant, J. M., A. M. O'Connor, I. R. Dohoo, H. N. Erb, M. Cevallos, M. Egger, A. K. Ersbøll, S. W. Martin, L. R. Nielsen, D. L. Pearl, D. U. Pfeiffer, J. Sanchez, M. E. Torrence, H. Vigre, C. Waldner, and M. P. Ward. 2016. Methods and processes of developing the strengthening the reporting of observational studies in epidemiology - veterinary (STROBE-Vet) statement. Prev. Vet. Med. 134:188-196. https://doi.org/10.1016/j.prevetmed.2016.09.005.

Sorge, U., D. Kelton, K. Lissemore, A. Godkin, S. Hendrick, and S. Wells. 2010. Attitudes of Canadian dairy farmers toward a voluntary Johne's disease control program. J. Dairy Sci. 93:1491-1499. https://doi.org/10.3168/jds.2009-2447.

Stafford, K. J., and D. J. Mellor. 2005. Dehorning and disbudding distress and its alleviation in calves. Vet. J. 169:337-349. https:// doi.org/10.1016/j.tvjl.2004.02.005.

Stafford, K. J., and D. J. Mellor. 2011. Addressing the pain associated with disbudding and dehorning in cattle. Appl. Anim. Behav. Sci. 135:226-231. https://doi.org/10.1016/j.applanim.2011.10.018.

Stilwell, G., R. C. de Carvalho, M. S. Lima, and D. M. Broom. 2009 Effect of caustic paste disbudding, using local anaesthesia with and without analgesia, on behaviour and cortisol of calves. Appl. Anim. Behav. Sci. 116:35-44. https://doi.org/10.1016/j.applanim 2008.06.008

Stilwell, G., R. C. Carvalho, N. Carolino, M. S. Lima, and D. M. Broom. 2010. Effect of hot-iron disbudding on behaviour and plasma cortisol of calves sedated with xylazine. Res. Vet. Sci. 88:188193. https://doi.org/10.1016/j.rvsc.2009.06.012.

Stock, M. L., S. L. Baldridge, D. Griffin, and J. F. Coetzee. 2013. Bovine dehorning: Assessing pain and providing analgesic management. Vet. Clin. North Am. Food Anim. Pract. 29:103-133. https: //doi.org/10.1016/j.cvfa.2012.11.001.

US Department of Agriculture. 2018. Dairy 2014, Health and management practices on U.S. dairy operations, 2014. USDA, USDAAPHIS-VS-CEAH-NAHMS. \#696.0218.
US Department of Agriculture. 2019. National Agricultural Statistics Service, 2017 Census of Agriculture. Dairy cattle and milk production. Accessed Apr. 18, 2021. www.nass.usda.gov/AgCensus.

Vasseur, E., F. Borderas, R. I. Cue, D. Lefebvre, D. Pellerin, J. Rushen, K. M. Wade, and A. M. de Passillé. 2010. A survey of dairy calf management practices in Canada that affect animal welfare. J. Dairy Sci. 93:1307-1315. https://doi.org/10.3168/jds.2009-2429.

Wikman, I., A. H. Hokkanen, M. Pastell, T. Kauppinen, A. Valros, and L. Hanninen. 2013. Dairy producer attitudes to pain in cattle in relation to disbudding calves. J. Dairy Sci. 96:6894-6903. https: //doi.org/10.3168/jds.2012-6128.

Winder, C. B., C. A. Bauman, T. F. Duffield, H. W. Barkema, G. P. Keefe, J. Dubuc, F. Uehlinger, and D. F. Kelton. 2018a. Canadian National Dairy Study: Heifer calf management. J. Dairy Sci. 101:10565-10579. https://doi.org/10.3168/jds.2018-14680.

Winder, C. B., S. J. LeBlanc, D. B. Haley, K. D. Lissemore, M. A Godkin, and T. F. Duffield. 2016. Practices for the disbudding and dehorning of dairy calves by veterinarians and dairy producers in Ontario, Canada. J. Dairy Sci. 99:10161-10173. https://doi.org/10 3168/jds.2016-11270.

Winder, C. B., S. J. LeBlanc, D. B. Haley, K. D. Lissemore, M. A. Godkin, and T. F. Duffield. 2017. Clinical trial of local anaesthetic protocols for acute pain associated with caustic paste disbudding in dairy calves. J. Dairy Sci. 100:6429-6441. https://doi.org/10 .3168/jds.2017-12724.

Winder, C. B., C. L. Miltenburg, J. M. Sargeant, S. J. LeBlanc, D. B Haley, K. D. Lissemore, M. A. Godkin, and T. F. Duffield. 2018b. Effects of local anesthetic or systemic analgesia on pain associated with cautery disbudding in calves: A systematic review and metaanalysis. J. Dairy Sci. 101:5411-5427. https://doi.org/10.3168/jds 2017-14092.

Wolf, C. A., G. T. Tonsor, M. G. S. McKendree, D. U. Thomson, and J. C. Swanson. 2016. Public and farmer perceptions of dairy cattle welfare in the United States. J. Dairy Sci. 99:5892-5903. https:// doi.org/10.3168/jds.2015-10619.

\section{ORCIDS}

Julia Saraceni ำ https://orcid.org/0000-0002-3661-6312

Charlotte B. Winder @ https://orcid.org/0000-0002-7314-3657

David L. Renaud (ㄴ https://orcid.org/0000-0002-3439-3987

Cynthia Miltenburg ๑ https://orcid.org/0000-0001-8945-6538

Erin Nelson @ https://orcid.org/0000-0002-7448-9588

Jennifer M. C. Van Os 우 https://orcid.org/0000-0002-7107-2867 